\title{
RADIAL DISTRIBUTIONS OF JULIA SETS OF MEROMORPHIC FUNCTIONS
}

\author{
LING QIU ${ }^{\varpi}$ and SHENGJIAN WU
}

(Received 15 July 2004; revised 18 July 2005)

Communicated by P. C. Fenton

\begin{abstract}
We consider a meromorphic function of finite lower order that has $\infty$ as its deficient value or as its Borel exceptional value. We prove that the set of limiting directions of its Julia set must have a definite range of measure.
\end{abstract}

2000 Mathematics subject classification: primary 30D45, 37F10.

\section{Introduction}

Let $f$ be a meromorphic function defined in the complex plane $\mathbb{C}$ or on the Riemann sphere $\overline{\mathbb{C}}=\mathbb{C} \cup\{\infty\}$. The Fatou set $F(f)$ of $f$ is the subset of $\overline{\mathbb{C}}$ where the iterates $f^{n}(n=1,2, \ldots)$ of $f$ are defined and $\left\{f^{n}\right\}$ forms a normal family. The complement of $F(f)$ is called the Julia set. It is obvious that $F(f)$ is an open set and $J(f)$ is closed. In general, the Julia set is very complicated.

Let $f(z)$ be a transcendental meromorphic function in the complex plane. Suppose that $\arg z=\theta$ is a ray from the origin. We say that $\theta$ is a limiting direction of $J(f)$ if, for any $\varepsilon>0$ and any $R>0$, the domain $\{z: \theta-\varepsilon<\arg z<\theta+\varepsilon,|z|>R\}$ has nonempty intersection with $J(f)$. We define the set $E \in[0,2 \pi)$ to be all the limiting directions of $J(f)$.

Baker first proved in [3] that, for a transcendental entire function $f$, the set $E$ contains infinitely many points. Later Qiao [6] proved that if the function is of finite lower order, then $E$ contains an interval whose length depends on the lower order.

Supported by the National Science Foundation of China (Grant No. 10171003 and 10231040) and the Doctoral Education Program Foundation of China.

(C) 2006 Australian Mathematical Society 1446-7887/06 $\$$ A2.00+0.00 
In [8], the authors considered the case of meromorphic functions with $\infty$ as their deficient value and, under some additional conditions, they proved the set $E$ has a definitely positive measure.

In this paper, we remove the additional condition in $[8$, Theorem 1$]$ and prove the following result.

THEOREM 1.1. Let $f(z)$ be a meromorphic function of lower order $\mu<\infty$ with deficiency $\delta(\infty, f)>0$. Then

$$
\operatorname{mes} E \geq \min \left\{2 \pi, \frac{4}{\mu} \arcsin \sqrt{\frac{\delta(\infty, f)}{2}}\right\} .
$$

If $\infty$ is a Borel exceptional value, then we can prove $E$ contains an interval with a definite length. Let $f(z)$ be a meromorphic function in $\mathbb{C}$ of order $0<\lambda<\infty$. Recall that $a \in \overline{\mathbb{C}}$ is a Borel exceptional value of $f(z)$ if it satisfies

$$
\varlimsup_{r \rightarrow \infty} \frac{\log n(r, f=a)}{\log r}<\lambda,
$$

where $n(r, f=a)$ is the counting function in value distribution theory of meromorphic functions.

In this case,we have the following result.

THEOREM 1.2. Let $f(z)$ be a transcendental meromorphic function of finite order $\lambda>0$. Suppose that $\infty$ is a Borel exceptional value of $f(z)$. Then there exists a closed interval $I \in R$ such that all $\theta \in I$ are limiting directions of $J(f)$ and $\operatorname{mes} I \geq \pi / \max (1 / 2, \lambda)$.

The proofs of the theorems depend strongly on the Nevanlinna theory of meromorphic functions. The reader can refer to [4] and [7] for the basic definitions and results in value distribution theory of meromorphic functions, in particular for the symbols such as $T(r, f), N(r, f)$, and so on.

\section{Proof of Theorems 1.1 and 1.2}

The following lemma, which is a special form of the result proved in [2], is sufficient to prove our theorem.

LEMMA 2.1 ([2]). Let $f(z)$ be a meromorphic function of finite lower order $\mu$. Suppose $\infty$ is a deficient value of $f$ with $\delta(\infty, f)>0$. Let $M_{j} \rightarrow+\infty(j \rightarrow \infty)$ and define

$$
E(r)=\left\{\theta:\left|f\left(r e^{i \theta}\right)\right|>r^{M_{j}}\right\}
$$


Then there is a sequence $\left\{r_{j}\right\}$ with $r_{j} \rightarrow \infty(j \rightarrow \infty)$ such that

$$
\liminf _{j \rightarrow \infty} \operatorname{mes} E\left(r_{j}\right) \geq \min \left\{2 \pi, \frac{4}{\mu} \arcsin \sqrt{\frac{\delta(\infty, f)}{2}}\right\} .
$$

In the following we denote the angular domain $\left\{z: \theta-\delta<\arg \left(z-z_{0}\right)<\theta+\delta\right\}$ by $\Omega\left(z_{0}, \theta, \delta\right)$, where $\theta \in \mathbb{R}$ and $0<\delta<\pi$. We state Lemma 1 from [6] in the following form.

LEMMA 2.2 ([6]). Let $f(z)$ be analytic in $\Omega\left(z_{0}, \theta, \delta\right)$. Suppose that $f\left(\Omega\left(z_{0}, \theta, \delta\right)\right)$ is contained in a simply connected hyperbolic domain in $\mathbb{C}$. Then

$$
|f(z)|<O(|z|)^{\pi / \delta}, \quad z \in \Omega\left(z_{0}, \theta, \delta^{\prime}\right)
$$

for any $\delta^{\prime} \in(0, \delta)$.

The proof of Lemma 2.2 is the same as that of [6, Lemma 1]. For meromorphic functions, the form we state in Lemma 2.2 is more convenient for our use.

PROOF OF THEOREM 1.1. Set

$$
\sigma=\min \left\{2 \pi, \frac{4}{\mu} \arcsin \sqrt{\frac{\delta(\infty, f)}{2}}\right\} .
$$

We conversely suppose that mes $E<\sigma$ and seek a contradiction.

Take a $t>0$ such that $\sigma-$ mes $E>t>0$. Since $E$ is closed, $S=[0,2 \pi) \backslash E$ consists of (at most countably many) open intervals $I$ from which we can find finitely many open intervals $I_{i}(i=1,2, \ldots, m)$ such that mes $\left(S \backslash \bigcup_{i=1}^{m} I_{i}\right)<K / 2$, where $K=\sigma-$ mes $E-t>0$. By the assumption of Theorem 1.1, it follows from Lemma 2.1 that there exists a sequence $\left\{r_{j}\right\}$ of positive numbers such that mes $E\left(r_{j}\right)>\sigma-t>0$, where $E\left(r_{j}\right)$ is defined as in (2.1). Obviously we have

$$
\operatorname{mes}\left(E\left(r_{j}\right) \cap S\right)=\operatorname{mes}\left(E\left(r_{j}\right) \backslash\left(E \cap E\left(r_{j}\right)\right)\right) \geq \operatorname{mes} E\left(r_{j}\right)-\operatorname{mes} E \geq K>0 .
$$

Thus there exists an open interval $I=I_{i_{0}} \subset S$ such that for infinitely many $j$

$$
\operatorname{mes}\left(E\left(r_{j}\right) \cap I\right)>\frac{K}{2 m}>0 .
$$

By passing to a subsequence if it is necessary, we can assume that for each $j,(2.2)$ holds. Write $I=(a, b)$. Take a positive number $\alpha$ such that

$$
\operatorname{mes}\left(E\left(r_{j}\right) \cap I_{\alpha}\right)>\frac{K}{3 m}>0, \quad j=1,2, \ldots,
$$


where we denote by $I_{\alpha}$ the interval $(a+\alpha, b-\alpha),(0<8 \alpha<b-a)$. It is easy to see from $I \cap E=\emptyset$ that there exists a positive $R$ such that

$$
\Omega\left(R, I_{\alpha}\right)=\left\{z \in \mathbb{C}:|z| \geq R \text { and } \arg z \in I_{\alpha}\right\} \subset F(f) .
$$

By choosing a point $z_{0}$ on the bisector of $I$, we see that the angular domain

$$
\left\{z: z \in \mathbb{C} ;\left|z-z_{0}\right| \geq 0 \text { and } \arg \left(z-z_{0}\right) \in I_{\alpha}\right\} \subset F(f) .
$$

So without loss of generality, we can suppose $\Omega\left(0, I_{\alpha}\right) \subset F(f)$.

In the following we assume that $\alpha$ is a fixed number such that (2.3) holds. Since $\Omega\left(0, I_{\alpha}\right) \subset F(f), f(z)$ has no pole in $\Omega$ and also does not take the values in $J(f)$. Take two fixed points $w_{j} \in J(f),(j=1,2)$. Thus $f$ is meromorphic in $\Omega\left(0, I_{\alpha}\right)$ and misses three points including infinity. Therefore the family $\{f \circ \varphi\}$, where $\varphi$ is a conformal automorphism of $\Omega\left(0, I_{\alpha}\right)$, is normal in $\Omega\left(0, I_{\alpha}\right)$ (compare [5]). So take a sequence of automorphisms $\varphi_{j}(z)$ of $\Omega\left(0, I_{\alpha}\right)$ such that $\varphi_{j}(z)=r_{j} z, r_{j}=\left|z_{j}\right|$. We see that $f \circ \varphi_{j}$ converges to a function $g$, which is either analytic or identically $\infty$ in $\Omega\left(0, I_{\alpha}\right)$. Now $f$ is unbounded on $\left\{z_{j}\right\}$ and hence $g \equiv \infty$. Thus $f \circ \varphi_{j}$ converges uniformly on $\{z:|z|=1\} \cap \Omega\left(0, I_{\alpha}\right)$ to $\infty$. This implies that

$$
\lim _{\substack{z \in L_{j} \\ j \rightarrow \infty}}|f(z)|=+\infty
$$

where $L_{j}=\left\{z:|z|=r_{j}\right\} \cap \Omega\left(0, I_{2 \alpha}\right)$.

In the following we prove the number of bounded components of $\mathbb{C} \backslash f\left(\Omega^{\prime}\right)$, where $\Omega^{\prime}=\Omega\left(0, I_{2 \alpha}\right)$ is at most one. If our conclusion is wrong, then we can take two bounded components $U_{1}, U_{2}$ from $\mathbb{C} \backslash f\left(\Omega^{\prime}\right)$. Choose two Jordan curves $\gamma_{1}, \gamma_{2}$ in $f\left(\Omega^{\prime}\right)$ such that $\gamma_{1}$ and $\gamma_{2}$ do not pass through critical values of $f(z), U_{1} \subset \operatorname{int}\left(\gamma_{1}\right)$, $U_{2} \subset \operatorname{int}\left(\gamma_{2}\right)$, and $\overline{\operatorname{int}\left(\gamma_{1}\right)} \cap \overline{\operatorname{int}\left(\gamma_{2}\right)}=\emptyset$. We choose a branch of $f^{-1}$ such that $f^{-1}\left(\gamma_{1}\right), f^{-1}\left(\gamma_{2}\right) \subset \Omega^{\prime}$. Then $f^{-1}\left(\gamma_{1}\right) \cap f^{-1}\left(\gamma_{2}\right)=\emptyset$. Take a fixed $R>0$ such that $\gamma_{1}, \gamma_{2} \subset\{z:|z|<R\}$. Noting that (2.4) holds, we see that every component of $f^{-1}\left(\gamma_{j}\right), j=1,2$, is bounded. Since the interior of $\gamma_{j}$ contains some points in $J(f)$, it is easy to see that any component of $f^{-1}\left(\gamma_{j}\right), j=1,2$, cannot be closed. So it is a Jordan arc. Now we take fixed $j_{0}$ such that $|f(z)|>R$ for all $z \in L_{j}\left(j>j_{0}\right)$ and $f^{-1}\left(\gamma_{j}\right) \cap \Omega^{\prime} \cap\left\{|z|<r_{j_{0}}\right\} \neq \emptyset, j=1,2$.

Take a component of $f^{-1}\left(\gamma_{j}\right), j=1$ or 2 , in $\Omega_{j_{0}}^{\prime}=\Omega^{\prime} \cap\left\{|z|<r_{j_{0}}\right\}$. Let $\sigma_{j}$ be a component of $f^{-1}\left(\gamma_{j}\right)$ in $\Omega_{j_{0}}^{\prime}, j=1,2$. It is easy to see that $\sigma_{1}$ is homotopic to $\sigma_{2}$. As $f(z)$ is analytic on $\bar{\Omega}_{j_{0}}^{\prime}$, we deduce that $\gamma_{1}=f\left(\sigma_{1}\right)$ is homotopic to $\gamma_{2}=f\left(\sigma_{2}\right)$. This is a contradiction, which proves our claim.

For a transcendental meromorphic function $f$, its Julia set is an unbounded set in $\mathbb{C}$. If $J(f)$ contains an unbounded component $\Gamma$, then $\mathbb{C} \backslash \Gamma$ is a simply connected hyperbolic domain $D$ and $f\left(\Omega^{\prime}\right) \subset D$. Otherwise all components of $J(f)$ are bounded 
and there are infinitely many bounded components in $J(f)$. Using the fact we just proved, it is not hard to find a simply connected hyperbolic domain $D \subset \mathbb{C}$ such that $f\left(\Omega^{\prime}\right) \subset D$.

Using Lemma 2.2, there exists a positive number $M$ such that $|f(z)|<|z|^{M}$ for all sufficiently large $z \in \Omega^{\prime}$. On the other hand, there are $z_{j} \in L_{j}$ such that $\left|f\left(z_{j}\right)\right|>$ $\left|z_{j}\right|^{M_{j}}$ for all sufficiently large $j$. Noting that $M_{j} \rightarrow \infty$, we get a contradiction and Theorem 1.1 is proved.

PROOF OF THEOREM 1.2. Let $f(z)$ be a transcendental meromorphic function in the complex domain of order $0<\lambda<\infty$. If $\infty$ is the Borel exceptional value of $f$, then

$$
\varlimsup_{r \rightarrow \infty} \frac{\log N(r, f)}{\log r}<\lambda
$$

Thus $f(z)$ must have the form $f(z)=G(z) / \Pi(z)$, where $G(z)$ is a transcendental entire function and $\Pi(z)$ is an entire function that is the typical product of the poles of $f(z)$. The functions $G(z)$ and $\Pi(z)$ have the following properties.

and

$$
\varlimsup_{r \rightarrow \infty} \frac{\log T(r, \Pi)}{\log r}=\varlimsup_{r \rightarrow \infty} \frac{\log m(r, \Pi)}{\log r}=\varlimsup_{r \rightarrow \infty} \frac{\log N(r, f)}{\log r}=\sigma<\lambda
$$

$$
\varlimsup_{r \rightarrow \infty} \frac{\log T(r, f)}{\log r}=\varlimsup_{r \rightarrow \infty} \frac{\log T(r, G)}{\log r}=\varlimsup_{r \rightarrow \infty} \frac{\log m(r, G)}{\log r}=\lambda .
$$

Since $G(z)$ is a transcendental entire function of finite order $\lambda$, it follows from the Phragmén-Lindelöf Theorem that there is an interval $(a, b)$ with $b-a \geq \min (2 \pi, \pi / \lambda)$ such that

$$
\limsup _{r \rightarrow \infty} \frac{\log \log \left|G\left(r e^{i \theta}\right)\right|}{\log r}=\lambda
$$

for all $\theta \in(a, b)$.

We are now able to prove $[a, b] \subset E$. If it is not true, then there is an subinterval $I \subset(a, b)$ such that the angular domain $\Omega(\{|z|>R, \arg z \in I\}) \subset F(f)$. Let $\arg z=\theta_{0}$ be the bisector of $I$. Then we have $\log \left|\Pi\left(r e^{i \theta_{0}}\right)\right|<r^{\sigma+\varepsilon}$, and

$$
\begin{aligned}
\log \left|f\left(r_{j} e^{i \theta_{0}}\right)\right| & =\log \left|\frac{G\left(r_{j} e^{i \theta_{0}}\right)}{\Pi\left(r_{j} e^{i \theta_{0}}\right)}\right|=\log \left|G\left(r_{j} e^{i \theta_{0}}\right)\right|-\log \left|\Pi\left(r_{j} e^{i \theta_{0}}\right)\right| \\
& >r_{j}^{\lambda-\varepsilon}-r_{j}^{\sigma+\varepsilon}=r_{j}^{\lambda-\varepsilon^{\prime}}
\end{aligned}
$$

for some $\varepsilon^{\prime}>0$. Thus we can find a sequences of points $\left\{z_{j}\right\}$ on the bisector such that $\log \left|f\left(z_{j}\right)\right|>\left|z_{j}\right|^{\lambda-\varepsilon}$ for some $\varepsilon>0$. 
Therefore, as in the proof of Theorem 1.1, we can find a sequence of

$$
L_{j}=\left\{\left|z_{j}\right| e^{i \theta}: a+\alpha \leq \theta \leq b-\alpha\right\}, \quad 0<\alpha<(b-a) / 8,
$$

such that (2.4) holds.

By the same argument of the proof of Theorem 1.1, we arrive at a contradiction. The proof of Theorem 1.2 is completed.

REMARK. Theorem 1.2 is also true for meromorphic functions of finite lower order $\mu$ with poles having order of growth less than $\mu$. In fact in this case, as in the proof of Theorem 1.2, $f$ can be written as $f(z)=G(z) / \Pi(z)$, where $G(z)$ is an entire function of finite lower order $\mu$, and $\Pi(z)$ is an entire function with order less than $\mu$. So applying a theorem of Baernstein in [1] to $G(z)$, we get a similar result as in Theorem 1.2.

\section{Acknowledgements}

We are grateful to the referee for suggestions which improved the presentation of the paper. In fact, the final remark is due to them.

\section{References}

[1] A. Baernstein, 'A generalization of the $\cos \pi \rho$ theorem', Trans. Amer. Math. Soc. 193 (1974), 181-197.

[2] —_ 'Proof of Edrei's spread conjecture', Proc. London Math. Soc. (3) 26 (1973), 418-434.

[3] I. N. Baker, 'Set of non-normality in iteration theory', J. London Math. Soc. 40 (1965), 499-502.

[4] W. K. Hayman, Meromorphic functions, Oxford Mathematical Monographs (Claredon Press, Oxford, 1964).

[5] O. Letho and K. Virtanen, 'Boundary behavior and normal meromorphic functions', Acta. Math. 97 (1957), 47-65.

[6] J.-Y. Qiao, 'On limiting directions of Julia sets', Ann. Acad. Sci. Fenn. Math. 26 (2001), 391-399.

[7] L. Yang, Value distribution theory, Translated from 1982 Chinese original (Springer-Verlag, Berlin; Science Press, Beijing, 1993).

[8] J.-H. Zheng, S. Wang and Z.-G. Huang, 'Some properties of Fatou and Julia set of transcendental meromorphic functions', Bull. Austral. Math. Soc. 66 (2002), 1-8.

\section{LMAM}

School of Mathematical Sciences

Peking University

Beijing 100871

P. R. China

e-mail: tangdin@math.pku.edu.cn 